\title{
Correction to: Proceedings of the 21st Congress of the International Ergonomics Association (IEA 2021)
}

Nancy L. Black, W. Patrick Neumann, and Ian Noy

Correction to:

N. L. Black et al. (Eds.): Proceedings of the 21st Congress

of the International Ergonomics Association (IEA 2021), LNNS 223, https://doi.org/10.1007/978-3-030-74614-8

In the original version of the book, the following belated corrections have been incorporated: The author name "Yue (Sophia) Li" has been changed to "Sofia Scataglini" in the part VI. 